Documentation et bibliothèques

DOCUMENTATION BIBLIOTHËQUES

\title{
Programme des fêtes de la Bibliothèque de l'Assemblée nationale : Deuxième centenaire
}

\section{Gaston Bernier}

Volume 47, numéro 2, avril-juin 2001

URI : https://id.erudit.org/iderudit/1032594ar

DOI : https://doi.org/10.7202/1032594ar

Aller au sommaire du numéro

\section{Éditeur(s)}

Association pour l'avancement des sciences et des techniques de la documentation (ASTED)

\section{ISSN}

0315-2340 (imprimé)

2291-8949 (numérique)

Découvrir la revue

\section{Citer cet article}

Bernier, G. (2001). Programme des fêtes de la Bibliothèque de l'Assemblée nationale : Deuxième centenaire. Documentation et bibliothèques, 47(2), 77-78. https://doi.org/10.7202/1032594ar

Tous droits réservés (C) Association pour l'avancement des sciences et des techniques de la documentation (ASTED), 2001
Ce document est protégé par la loi sur le droit d'auteur. L'utilisation des services d'Érudit (y compris la reproduction) est assujettie à sa politique d'utilisation que vous pouvez consulter en ligne.

https://apropos.erudit.org/fr/usagers/politique-dutilisation/ 


\title{
Programme des fêtes de la Bibliothèque de l'Assemblée nationale: Deuxième centenaire
}

\author{
Gaston Bernier \\ Coordonnateur du Deuxième centenaire \\ Bibliothèque de l'Assemblée nationale du Québec
}

La Bibliothèque de l'Assemblée nationale marquera l'anniversaire de ses deux cents ans en 2002. Le premier centenaire, en 1902, est passé inaperçu, mais on peut croire cependant que le conservateur de l'époque, N.E. Dionne, voulut le souligner ainsi que celui de la naissance des institutions parlementaires en publiant un historique de la bibliothèque cette année-là.

L'organisation de la commémoration s'insère assez naturellement dans le sillage d'activités antérieures similaires comme celles signalant la loi accordant des droits politiques aux personnes de religion juive (1982) et celles du Barreau du Québec (1999), le cinquantième du droit de vote des femmes (1990) et de la création d'Hydro-Québec (1994), le bicentenaire des institutions parlementaires en 1992, le cent vingt-cinquième anniversaire de la Tribune de la presse en 1996, le centième de la première séance tenue dans l'actuel palais législatif, le quatre-vingtième du Parlementaire (1997), le trentecinquième du Journal des débats (1999).

Jusqu'à maintenant, le projet de célébration n'a pas été entériné de manière formelle par les autorités politiques, c'est-àdire par les membres du Bureau de l'Assemblée. Ces derniers avaient préalablement pris connaissance, en juin 2000 , d'un court mémoire portant sur le sujet et donnant les grandes lignes des activités envisagées, de leurs objectifs, des montants nécessaires et de la gestion de l'ensemble. Par ailleurs, un texte plus substantiel avait été préparé au printemps 1999 à la demande du secrétaire général et lui a été acheminé le 5 mars 1999. Les membres du Bureau ont reçu le coordonnateur du Deuxième centenaire le 28 mars dernier et lui ont demandé de présenter un programme. Les membres ont accueilli favorablement les projets présentés.
Rappelons que les députés ont, de fait, établi leur première bibliothèque le 10 mars 1802: ce jour-là, ils nommèrent les membres d'un comité chargé de gérer les livres réceptionnés depuis quelques mois, l'achat avait été approuvé un an plus tôt, et d'élaborer les premiers éléments d'un règlement interne. C'est entre 1792 et 1802 que les parlementaires utilisèrent une bibliothèque privée créée en 1779 à l'initiative du gouverneur Haldimand et logée dans le Palais épiscopal, siège de la Chambre basse et de la Chambre haute. L'assemblée des propriétaires avait accepté, le 29 décembre 1792, «que les Membres de la législature, non souscripteurs et qui ne résident pas à Québec auront l'usage des livres pendant la session [...] en payant trois piastres et se conformant aux règles " (La Gazette de Québec, 3 janvier 1793). À ce moment-là, la Bibliothèque de Québec possédait 2443 volumes, dont 1211 en anglais, 1209 en français, et 23 en latin et en grec et, à l'initiative du conservateur, on en avait déjà publié un catalogue (Le Boréal express, 1760-1810, p. 369). Le 13 janvier 1802, l'Orateur informe la Chambre que «la plus grande partie des livres votés [...] l'année dernière était arrivée et était sous la garde du Greffier...» (Journal de la Chambre..., 1802, p. 27).

\section{Objectifs de la célébration}

Les manifestations prévues en vue de l'anniversaire sont essentiellement destinées à mettre en exergue la vision et l'esprit d'ouverture des présidents et dirigeants politiques tant du $\mathrm{XIX}^{\mathrm{e}}$ que du $\mathrm{X} \mathrm{X}^{\mathrm{e}}$ siècle, à intensifier les relations avec les parlementaires actuels et futurs, avec leurs collaborateurs, à raffermir les contacts avec les fonctionnaires du secrétariat administratif, à rendre hommage à l'effectif documentaire en place et aux prédécesseurs et, enfin, à souligner les liens avec les homologues bibliothécaires et les autres bibliothèques des réseaux documentaires formels ou officieux.

\section{Activités envisagées}

Le programme de commémoration se déroulera surtout au cours des douze mois de 2002. Cependant, il y aura des réalisations en 2001 destinées à annoncer l'anniversaire et à sensibiliser le milieu : publication d'un recueil de textes des années 1802 à 2002, d'un calendrier historique identifiant les activités du Deuxième centenaire, d'une livraison spéciale du Bulletin de la Bibliothèque, des biographies des conservateurs, de textes publicitaires dans des revues professionnelles et dans des publications de l'Assemblée, dessin et dévoilement d'un emblème ou logotype. Précédemment, il y aura eu l'actualisation et la publication, en mars 2000 , de la brochure La Bibliothèque...; bientôt 200 ans : 1802-2002; l'éclairage du vitrail, en automne 2000 , la proposition de classement de la collection du premier ministre Chauveau conservée à la Bibliothèque et adressée à madame la ministre de la Culture et des Communications en mai 1999.

L'anniversaire proprement dit sera marqué par des activités de nature protocolaire si les autorités donnent leur aval: une cérémonie d'ouverture en mars 2002 et une de clôture en décembre, le dévoilement d'une mosaïque des conservateurs en poste dans le passé et d'une autre des employés du moment, peut-être d'une médaille souvenir, d'une plaque commémorative suggérée à la Commis- 
sion de la capitale nationale et d'un timbre-poste.

Côté professionnel, il y aura, entre autres, la rencontre bisannuelle de l'Association des bibliothécaires parlementaires du Canada (APLIC ou ABPAC) en août ou septembre 2002. Ce sera la quinzième rencontre depuis sa création dans les années 1970 et la troisième à Québec. L'Assemblée nationale en avait été l'hôte en 1978 et en 1992. On tâchera, pour l'occasion de préparer un programme spécial. De plus, le congrès devrait être précédé ou suivi d'un colloque consacré à l'histoire des bibliothèques parlementaires. On prévoit également organiser quatre déjeunersconférences en cours d'année, probablement au restaurant parlementaire: les conférenciers seront un bibliothécaire parlementaire étranger, des personnalités politiques, en fonction ou retraitées, des hauts fonctionnaires ou des universitaires. À compter de la fin de janvier, on pourra voir une exposition portant sur «les conservateurs de la Bibliothèque " et sur l'apport de leurs patrons, les présidents.

Le programme de publication inauguré en 2001 se poursuivra lors de l'année anniversaire. On prévoit la rédaction d'une histoire de la Bibliothèque, d'un essai sur la collection composée entre 1802 et 1849 , année d'un premier incendie, d'une livraison spéciale de Documentation et bibliothèques consacrée aux bibliothèques parlementaires, à leur histoire, à leur gestion, à leurs fonctions, à leurs collections, à leur lectorat et à leur avenir, une livraison du Bulletin de la Bibliothèque de l'Assemblée... et, enfin, d'un testimonial constitué de témoignages de lecteurs contemporains et anciens. On aimerait bien également réaliser un cédérom des projets de loi présentés à l'Assemblée entre 1868 et 1930.

Enfin, dernier volet, on prévoit des activités de nature sociale et publicitaire. On réalisera une affiche du vitrail de Guido Nincheri ornant la Bibliothèque et illustrant la pérennité de la science. Des journées «portes ouvertes " à l'intention des familles des employés en place, pour les retraités et pour les parlementaires, leurs colla- borateurs et le personnel administratif au sens large. Par ailleurs, on a suggéré que la Bibliothèque soit au programme des visites guidées pour l'année du Deuxième centenaire. On produira également d'autres articles publicitaires: cartes postales, signets, épinglettes, etc. Un bulletin d'information sera publié bimestriellement d'ici à 2002. Une première livraison fut distribuée en janvier 2001, une deuxième, en mars.

D'autres entreprises ont été envisagées: la rédaction d'un dictionnaire des institutions parlementaires, une livraison spéciale d'une revue de presse historique, le regroupement des services de la Bibliothèque, la réalisation d'un casse-tête reproduisant le vitrail de Nincheri.

\section{Le numéraire}

On évalue à 60000 \$ le coût des célébrations envisagées. Beaucoup d'activités seront réalisées à même les crédits ordinaires de la Bibliothèque ou des crédits des différents services de l'Assemblée nationale.

Idéalement, les projets devraient être financés à même les crédits habituels et le plus grand nombre possible sera pris en charge par les services internes (Direction des communications, de l'accueil, imprimerie, etc.) ; des commandites seront sollicitées auprès d'associations professionnelles, de fournisseurs de la Bibliothèque et d'autres entreprises, si une telle initiative ne contrevient pas aux pratiques de l'Assemblée nationale. Par ailleurs, une certaine forme de bénévolat est mise à contribution: au cours de l'automne, neuf étudiants du département d'histoire de l'Université Laval ont entrepris des travaux de repérage de textes, d'analyse de la collection historique, de rédaction de biographies, etc.

\section{L'organisation}

Pour l'heure, la planification et l'organisation de la célébration du Deuxième centenaire sont de la compétence d'un comité formé d'employés et de retraités de la Bibliothèque, d'un représentant de la Direction des études et d'un autre des communications.

Antérieurement, un premier comité fut chargé de dresser un inventaire des activités possibles. II siégea à quatre reprises et remit son rapport au Comité de coordination de la Bibliothèque en février 1999.

Les membres du comité d'organisation ont proposé, pour l'année anniversaire, la composition de deux comités : l'un dit d'honneur, formé d'hommes politiques et d'anciens conservateurs (ou de leurs descendants) et un Comité directeur, formé du président de l'Assemblée, de hauts fonctionnaires parlementaires et du coordonnateur du deuxième centenaire. Les activités seraient tenues sous le haut patronage de celui-là; à celui-ci reviendraient la coordination générale et le contrôle.

\section{Conclusion}

En somme, les activités proposées à l'occasion du Deuxième centenaire porteront avant tout la marque de l'établissement documentaire et de l'Assemblée nationale elle-même. II y aura un minimum de réceptions, de brindisis et de rencontres sociales, de messages publicitaires et de «feux d'artifice». Les entreprises suggérées devraient, dans l'ensemble, laisser des marques ou des traces et constituer sinon un tremplin du moins un marchepied pour la relève et les successeurs, marquer l'entrée toute récente, encore à ce moment-là, dans le $\left.X X\right|^{e}$ siècle et indiquer une direction dans l'évolution des services documentaires que la représentation nationale se donne et dont elle ressent un besoin quasi impérieux. 